\title{
Capsule Commentary on Deliz et al., Cultural Competency Interventions during Medical School: a Scoping Review and Narrative Synthesis
}

\author{
Zach Affeldt, MS
}

Medical College of Wisconsin, Milwaukee, WI, USA.

J Gen Intern Med 35(2):623

DOI: $10.1007 /$ s11606-019-05547-w

(c) Society of General Internal Medicine 2019

$\mathrm{P}$ hysicians with cultural competence training have greater patient satisfaction. ${ }^{1}$ Consequently, many medical schools strive to instill cultural competence in their students. However, the best approach to teaching cultural competence remains difficult to assess due to difficulties assessing the effects of individual interventions and their outcomes. ${ }^{2}$ Additional issues include that many existing cultural competency interventions fail to address implicit biases students may hold and many institutions do not provide cultural training for their faculty. ${ }^{3}$

This Meta-analysis by Deliz et al. ${ }^{4}$ summarizes the current cultural competency teaching for medical students and their evaluation methods. They included 154 articles on cultural competency with 56 having a general focus and 98 focusing on specific populations that include race/ethnicity, global health, socioeconomic status, language, immigration status, disability, spirituality at the end of life, rurality, and LGBTQ. Most of these interventions (53.9\%) used lectures as the instructional method; the second most popular method was discussion (36.4\%). Most studies evaluated (90.3\%) the impact of this training using surveys or knowledge-based tests. Most interventions were brief ( $1-3 \mathrm{~h})$, though some were longitudinal throughout the students' medical education. The authors concluded that the lack of a standardized way to objectively assess the effect of interventions on patient and physician outcomes limited institutions' ability to make informed decisions on how to structure a culture competency curriculum.

Published online November 21, 2019
For institutions and administrators, this study identifies the current practices of medical schools in curricula pertaining to cultural competency. While unable to identify the best approach, this study provides a framework for curriculum developers to use. Additional studies, improved and objective outcome assessment and, ideally, randomized trials comparing different instruction methods will be required in order to fully understand how to best groom culturally competent medical students.

Corresponding Author: Zach Affeldt, MS; Medical College of Wisconsin, Milwaukee, WI, USA (e-mail: Zaffeldt@mcw.edu).

Compliance with Ethical Standards:

Conflict of Interest: The author declares that he/she does not have a conflict of interest.

\section{REFERENCES}

1. Alizadeh S, Chavan, M. Cultural Competence Dimensions and Outcomes: A Systematic Review of the Literature. Health and Social Care In The Community. 2015; 24(6): 117-130

2. Jernigan VB, Hearod JB, Tran $\mathbf{K}$, Norris KC, Buchwald D. An Examination of Cultural Competence Training in US Medical Education Guided by the Tool for Assessing Cultural Competence Training. J Health Dispar Res Pract. 2016;9(3):150-167.

3. Sorensen J, Norredam M, Dogra N, Essink-Bot ML, Suurmond J, Krasnik A. Enhancing cultural competence in medical education. Int $J$ Med Educ. 2017;8:28-30. Published 2017 Jan 26. https://doi.org/10. 5116/ijme.587a.0333

4. Deliz JR, Fears FF, Jibe Jem Taibat Hm Cgar Dm Riss WR. Cultural competency interventions during medical school: A scoping review and narrative synthesis. J Gen Intern Med (SPI 5417.)

Publisher's Note Springer Nature remains neutral with regard to jurisdictional claims in published maps and institutional affiliations. 